\title{
Exploring a broad spectrum of design options for DEMO
}

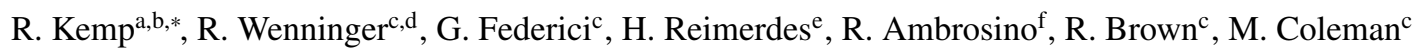 \\ ${ }^{a}$ CCFE, Culham Science Centre, Oxfordshire OX14 3DB, United Kingdom \\ ${ }^{b}$ EUROfusion PMU, Culham Science Centre, Oxfordshire OX14 3DB, United Kingdom \\ ${ }^{c}$ EUROfusion PMU, Boltzmannstr.2, Garching 85748, Germany \\ ${ }^{d} I P P$, Boltzmannstr.2, Garching 85748, Germany \\ ${ }^{e}$ EPFL, CH-1015 Lausanne, Switzerland \\ ${ }^{f}$ Università di Napoli Parthenope, Via Medina, 40, 80133 Napoli, Italy
}

\begin{abstract}
In the pursuit of realistically achievable design options for demonstrating fusion electricity generation and tritium self-sufficiency in a device to follow ITER, it is vital to explore as fully as possible the available design space and technology options which might lead to a fusion power plant within the timescales envisioned by the EU Roadmap to Fusion Energy. The usual tool for exploring this space is a systems code, such as PROCESS, which seeks to model all important plant systems and physics to provide an integrated power plant design point. However, currently many of these models are tied to assumptions of ITER-like technology and therefore tend to lead to ITER-like plant solutions.

This contribution describes a broader set of plant configurations being considered alongside the main baseline design, investigating the impacts on design and costs of designing for (1) flexi-pulsed-steady-state operation, (2) double-null divertors, and (3) the use of high-temperature superconductors. The focus of the work presented here, however, is on (4) advanced magnetic configurations such as snowflake and super-X divertors. We discuss the modifications necessary for the systems code to simulate these configurations and their performance, particularly the divertor geometry and power handling capability; rapid engineering analysis of TF and PF coil positions which can achieve both the required equilibrium and remote-handling access; and initial wider analysis of the physics, neutronics, and other considerations. The reductions in wall area available for breeding tritium affect the choice of blanket technology, and remote handling considerations have a strong impact on the configurations which can be considered reasonable from an engineering and availability perspective. The benefits, disadvantages, risks, and power plant relevance of each configuration over the baseline DEMO design are discussed.
\end{abstract}

Keywords: DEMO, systems studies, system modelling, fusion power plant, technology choices

\section{Introduction}

The aim of the EUROfusion research programme is to develop the technology and physics basis for commercial fusion power. In order to develop the systems to achieve this goal, EUROfusion is designing a demonstration fusion power plant, termed DEMO. The goals of this project are to achieve tritium self-sufficiency; provide substantial power to the grid; and demonstrate supporting technologies and strategies for future power plants, for example fully-operational remote handling and maintenance systems, data to satisfy industry regulators, and so on. The lowest-risk approach is to follow the path laid by ITER and make ITER-like assumptions for physics and technological performance, and this is the approach taken by the nominal EUROfusion baseline design. However, it is also valuable to consider variations on this design using alternative technologies and physics assumptions which may provide useful information on research directions or design variations which would help to increase confidence in DEMO achieving its design goals, or re-

\footnotetext{
${ }^{*}$ Corresponding author

Email address: richard.kemp@ukaea.uk (R. Kemp)
}

duce the necessary steps beyond DEMO to fully commercial and economically competitive fusion power.

The EUROfusion roadmap [1] targets the production of electricity from fusion in the 2050s. This implies that building work on DEMO must begin before 2040 with most final design decisions made well in advance of this to allow the completion of engineering design work. Technology choices must be made before this so that systems integration can be completed and requirements set. Overall, the conclusion is that in order to meet the roadmap target, we need technologies that exist today, at least in functional form, to allow downselection and further development to the required levels. We cannot rely on the delivery of breakthrough technologies that will slot into plant designs predicated on their existence.

To assist the technology and plant layout downselection and guide where research investment may be most fruitful, a number of alternative DEMO architectures and their sub-variants were examined in parallel so that a well-informed assessment of options, driven by a rigorous systems engineering approach and plant assessment criteria, can be carried out. The purpose of these alternative architecture studies is twofold. First, they should be defined to cover a wider solution space and stimulate 
the development of alternatives which perform better against the plant assessment criteria. In particular these should address major uncertainties and shortcomings identified in the baseline (e.g., improved concept of power exhaust and associated first wall protection strategies - recognised to be one of the most crucial interfaces for the design and development of fusion power systems), or investigate upfront potential showstoppers linked to the adoption of specific design choices affecting safety and/or the overall plant layout and operation.

\section{DEMO design options}

The 2017 DEMO baseline is summarised in Figure 1. It assumes modest advances on ITER physics and technology, with a target of 500MW net electrical power and a minimum pulse length of 2 hours. The alternative architectures are as follows:

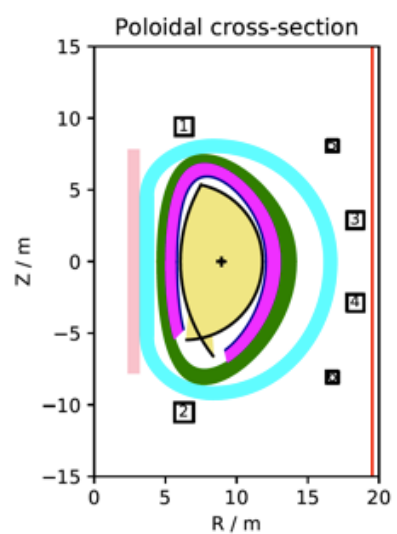

\begin{tabular}{|l|l|}
\hline Characteristic & Value \\
\hline$R_{0} / a(\mathrm{~m})$ & $8.9 / 2.9$ \\
$\kappa_{95} / \delta_{95}$ & $1.65 / 0.33$ \\
Fusion power $(\mathrm{MW})$ & 1998 \\
Burn time $(\mathrm{s})$ & 7200 \\
$\beta_{\mathrm{N}, \mathrm{tot}}$ & 2.9 \\
$P_{\mathrm{sep}} B /\left(q A R_{0}\right)(\mathrm{MW} \mathrm{T} / \mathrm{m})$ & 9.2 \\
\hline
\end{tabular}

Figure 1: Key parameters from the 2017 EU DEMO baseline. The final value is the divertor challenge quantifier.

\subsection{Flexi-DEMO}

Flexi-DEMO refers to a variant of DEMO where the initial design is based around higher-performance physics and additional heating/current drive power, similar to the scenario expected from the steady-state ITER $Q=5$ Scenario [2]. The design has then been iterated to allow the recovery of operational performance should the scenario fall short of these targets, by operating in pulsed mode rather than steady-state. This option requires confidence in physics extrapolation and highly-reliable and efficient current-drive systems [3].

\subsection{Double-null}

An issue with the conventional single-divertor DEMO design is the power loading around the secondary X-point at the top of the plasma, potentially requiring the use of limiters, which occlude sections of the blanket, reducing tritium breeding and electricity generation. This can be overcome by the use of a double-null design, although obviously with additional complications to the remote handling and therefore potential overheads for the availability. The upper divertor also removes sections of the breeder blanket. Whether or not the tritium breeding ratio (TBR) is more reduced by the use of limiters or the double-null configuration depends on design details which are still uncertain. There are on the other hand some additional potential benefits, for example the reduction of load to the inner divertor leg may allow the incorporation of these strike points into the blanket segments.

\subsection{High-temperature superconductors (HTS)}

High-temperature superconductors potentially allow the use of high magnetic fields when operating at low (liquid He) temperature. However forces in the TF coils vary as $B^{2}$ and so achieving high fields in practice is also limited by the structural materials available. However it is worth investigating what might be achievable. Other benefits (not reviewed as part of this work) may include demountable (or remotely manufacturable) coils; and higher flux swing if used in the CS (although this may also require stronger materials) [4]. High temperature (liquid $\mathrm{N}$ ) operation is still seen as very ambitious.

\subsection{Advanced magnetic configurations (AMC)}

These options refer to a family of divertor configurations, including super-X [5], snowflake [6], and long leg [7]. They have been explored within the EUROfusion DTT1 project [8] by expansion around the extant baseline to confirm that the configurations could be achieved. However, the outcomes of this work need to be re-integrated into a complete plant design system to explore the costs and benefits of incorporating each option into a power plant. The work presented here focusses on these options.

\section{AMC operating point analysis}

The analysis was developed as a rapid-assessment workflow for DEMO options. This attempted to identify and then rule out or otherwise circumvent any potential showstoppers, such as the requirement for PF coils in locations inhibiting remote maintenance access or unacceptable loss of tritium breeding capability. The major steps are outlined below.

\subsection{Achieving an equilibrium}

The basic requirements for achieving an equilibrium for each AMC were first of all assessed, including the shaping $(\kappa$ and $\delta$ ) and resulting plasma volume, and effects on plasma current. The requirements on divertor space were also assessed. This allowed a first estimate of overall machine geometry and build for a systems code. 


\subsection{Systems codes}

The fusion power plant systems code PROCESS [9] was used to find integrated machine designs that met the high-level performance required of EU-DEMO: a net electrical power of $500 \mathrm{MW}$ and burn time of 2 hours, whilst minimising the device major radius. Changes were made to the existing (2017) baseline to reflect the differences identified in the equilibrium work. In addition, it was found that the inclusion of the PF currents necessary to produce a snowflake configuration reduced the available flux swing from the CS: this was partially overcome by re-optimisation of the CS (Figure 2) but was still lower than the standard divertor configuration. Factors covering the occlusion of the first wall by limiters, and the neutron loss to the divertor were introduced, and the space required for the divertor inside the TF coil in each case was set. These values are summarised in table 1.

\subsection{Magnet engineering}

A code for assessing optimised coil configurations, NOVA, was used to identify minimal PF coil sets which still permitted RH access. The operation and results of this code have been described elsewhere [7]. Limiting factors on the coil layout include stress limits and current densities as well as magnetic field design.

\subsection{Remote handling (RH)}

As well as a PF coilset allowing remote handling access, the divertor cassette must also be of handleable size and weight. The cutting and reconnection of services to in-vessel components is a major RH overhead [10] and designs which lengthen or complicate this process are not preferred. This counts most strongly against in-vessel coils of any design which may require many services and connections per port.

\subsection{Other considerations}

Since there are no reliable predictive models for the performance of particular divertor configurations, a range of $\frac{P_{\text {sep }}}{R_{0}}$ values was explored to identify performance targets for each configuration, at which they would provide global benefits to the device construction. Further specialised modelling and experiments can then be conducted to see if the configurations are capable of meeting these targets.

\begin{tabular}{|l|c|c|c|c|}
\hline Parameter & ITER-like SN & DN & SF SN & SX DN \\
\hline$f_{\mathrm{HGH}}$ & 0.91 & 0.94 & 0.9 & 0.94 \\
$h_{\text {div }}(\mathrm{m})$ & 0.621 & 0.621 & 0.932 & 1.2 \\
$\Phi$ & - & - & $-25 \%$ & - \\
$\delta_{95}$ & 0.33 & 0.33 & 0.25 & 0.33 \\
\hline
\end{tabular}

Table 1: Main differences in input configurations for systems code. $\mathrm{SN}=$ single-null; $\mathrm{DN}=$ double-null; $\mathrm{SF}=$ snowflake and $\mathrm{SX}=$ super-X. $f_{\mathrm{HGH}}=$ first-wall area not occupied by limiters or divertor, available for recover of highgrade heat and tritium breeding. $h_{\text {div }}=$ height of divertor structure below X-pt; the SX leg is also elongated. $\Phi$ is change in available flux swing due to CS reconfiguration.
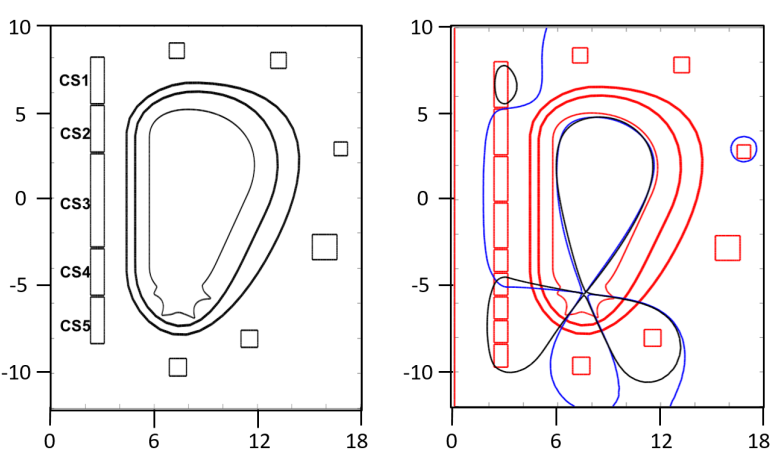

Figure 2: (left) Initial DEMO CS segmentation and (right) optimised segmentation of CS to recover flux swing. This results in a $33 \%$ increase in flux swing with a snowflake configuration over the original configuration. However the plasma triangularity is reduced from 0.33 (ITER-like single null, at the $95 \%$ flux surface) to 0.25 . Scales are in metres.

\section{Results}

\subsection{Super-X and long-leg}

Since the purpose of these configurations is to spread the power from the outer divertor leg over a larger area through flux spreading and radiation, their impact is limited in single-null configurations due to the high heat loads expected at the inner leg. If the inner divertor plate is close to its limits, more power cannot be put into the SoL without overloading it, regardless of the capabilities of the outer leg, unless methods are identified of varying the inner-outer power split. Therefore these solutions are suitable for double-null configurations only, substantially increasing the magnetised volume of the machine (table 3). In the case of a super- $X$ configuration, coils are also required inside the TF coils. Further work would be required to evaluate if these could be made discontinuous (as in e.g. [5]) and incorporated into divertor cassettes with reasonable lifetimes and cryogenic performance before this configuration can be seriously considered as power-plant relevant. Further issues which must be resolved, not fully evaluated here, include potentially complex TF coil shapes to optimise unused magnetised volume, with accompanying increase in forces and costs. Plasma control to maintain the divertor configuration is also an underexplored area and almost certainly presents greater challenges than conventional (single- and double-null) short leg divertors.

\subsection{Snowflake}

The principal benefit of a snowflake configuration appears to be the ability to radiate high powers stably from the X-point region, avoiding the inner-leg loading problem of super-X and long-leg. These configurations are also achievable using exvessel coil configurations which preserve RH access to the vessel. However, they occupy more volume and the reduced plasma shaping and flux swing from the CS coil mean that SoL radiation has to be significantly higher than from a conventional divertor in order to achieve a similar overall device size. In addition, the increased radiation means much higher first-wall radiation loads in the area of the divertor throat (table 2). Even 


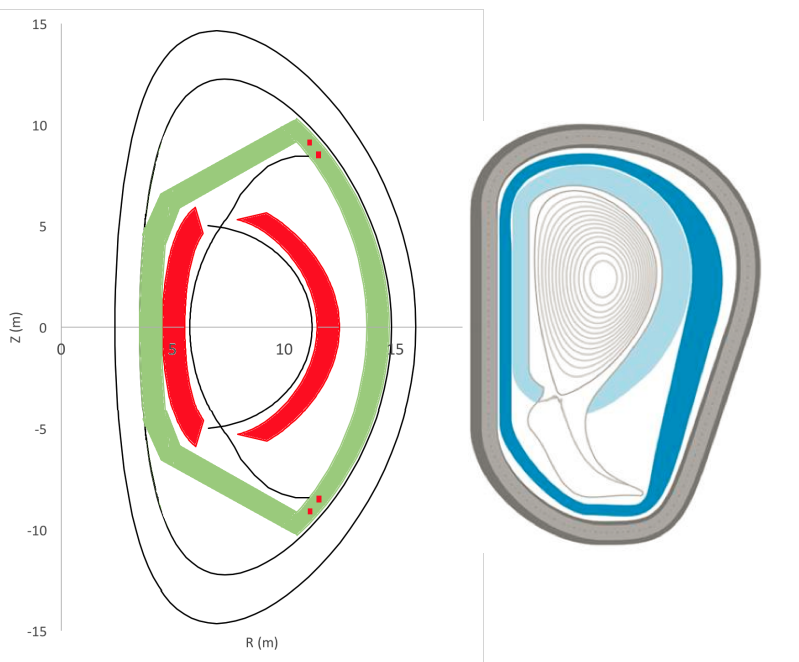

\begin{tabular}{|l|c|c|c|}
\hline Parameter & ITER-like SN & SX DN1 & SX DN2 \\
\hline$R_{0}(\mathrm{~m})$ & 8.9 & 8.9 & 8.5 \\
TF h/w $(\mathrm{m})$ & $18.4 / 14.1$ & $27.2 / 13.8$ & $26.7 / 13.5$ \\
$E_{\text {mag }}(\mathrm{GJ})$ & 141.4 & 224.9 & 207.6 \\
TF mass $(\mathrm{kt})$ & 17.8 & 24.6 & 23.0 \\
$P_{\text {divt }}(\mathrm{MW})$ & 155 & 149 & 274 \\
$f_{\mathrm{LH}}$ & 1.4 & 1.4 & 2.6 \\
\hline
\end{tabular}

Figure 3: Results of accommodating a super-X divertor in a double-null configuration in PROCESS (left). PROCESS assumes D-shaped coils which must accommodate the extended divertor leg. SX DN1 assumes no improvement in divertor capabilities. For SX DN2, assuming a $\frac{1}{3}: \frac{2}{3}$ inner/outer power split to the divertor legs, at most this configuration can double the divertor capabilities. This allows some reduction in major radius but the stored magnetic energy (as a proxy for TF coil costs) are still higher than the ITER-like case (TF h/w refers to TF height and width). The systems code enlarges the TF coil to ensure the long SX limbs have sufficient space. It is possible that the TF coil shapes could be optimised to reduce this but at the cost of introducing stress-multiplying small-radius corners and more complex windings (right: DTT1 output for a single-null SX divertor minimising the magnetised volume, with conspicuously square TF coil). In addition, this now requires $100 \mathrm{MW}$ to be stably radiated from each outer divertor leg (resulting in an estimated 0.2$0.3 \mathrm{MW} / \mathrm{m}$ on the divertor surfaces). Other engineering objections are given in the text. Components in plot, from plasma centre: plasma; breeder blanket; shield/vacuum vessel; TF coil. in this case, the larger divertor volume means that the stored magnetic energy is around $10 \%$ higher than the baseline, implying additional capital cost. However, if the overall system reliability is higher (the lower core plasma radiation fraction and operation well above the LH transition implies a more stable plasma) and impacts on the RH schedule are not onerous, this additional cost will be overcome in operations benefits.

\begin{tabular}{|l|c|c|c|c|c|}
\hline & SN & DN & DN HR & SF SN1 & SF SN2 \\
\hline$R_{0}$ & 8.9 & 8.7 & 8.6 & 9.3 & 8.9 \\
$E_{\mathrm{mag}}$ & 141.4 & 151.3 & 142.0 & 166.0 & 155.0 \\
$f_{\mathrm{LH}}$ & 1.4 & 1.7 & 2.0 & 1.4 & 2.2 \\
$f_{\mathrm{rad}}$ & 0.63 & 0.57 & 0.48 & 0.62 & 0.44 \\
$P_{\mathrm{rad}}$ & 110 & $110 / 55$ & $150 / 75$ & 118 & 247 \\
$\Gamma_{\mathrm{rad}, \max }$ & 0.6 & 0.3 & 0.4 & 0.64 & 1.1 \\
$\frac{P_{\mathrm{sep}} B}{q A R_{0}}$ & 9.2 & 10.8 & 13.5 & 9.2 & 14.6 \\
\hline
\end{tabular}

Table 2: Comparison of solutions for double-null and snowflake to the baseline (SN). The double null solutions are assuming no particular improvement in radiation but power split between four divertor plates (DN), and for extra radiation at the second X-point (DN HR, $P_{\text {rad }}$ is in MW, total and per X-point). SF $\mathrm{SN} 1$ is a snowflake configuration for no improvement in $\mathrm{X}$-point radiation, and SF SN2 has the performance $\frac{P_{\mathrm{sep}} B}{q A R_{0}}\left(\mathrm{MW} \mathrm{T} \mathrm{m}{ }^{-1}\right)$ value for which the machine returns to the $\mathrm{SN}$ baseline dimensions (although stored magnetic energy, $E_{\mathrm{mag}}$ $(\mathrm{GJ})$, is still higher to accommodate the larger divertor. $\Gamma_{\text {rad,max }}\left(\mathrm{MW} \mathrm{m}^{-2}\right)$ is the estimated radiative load on the first wall close to the X-point; for SF SN2 this is close to material limits.

\section{Power plant relevance}

Two of the principal factors required to allow fusion electricity to be economically competitive are lower capital cost and high availability (implying also high reliability). As a first of a kind plant, DEMO must demonstrate technology capable of achieving both of these. The latter means that efficient remote handling must be possible, components must have good expected lifetimes, and plasma scenarios must be stable and have good operating margins. Ideally DEMO will also have options for flexibility to allow development and testing of materials and components which will not be ready for the base DEMO scenario but still require in-machine qualification before they can be used as the basis of plant design. However, the choice of divertor geometry, major operating scenario or superconductor cannot be changed during operation and so in these areas DEMO must either itself be power-plant relevant (that is, there must be a path from DEMO technologies to develop to the performance required of those technologies in a power plant) or there must be a parallel research path to develop and qualify those technologies. The exploration and assessment of this design space and these options allows identification of potential research opportunities and, conversely, areas where breakthroughs are required before options can be seriously considered and so downselection is appropriate within the EUROfusion programme. 


\section{Alternative concept development}

This work also points towards a process for rapid evaluation of proposed alternative technologies and concepts. When a new technology is proposed its benefits can be outlined but the aspects of integrating it into a power plant design - for example here, changes to the CS, plasma shaping, divertor dimensions, and so on - must be identified so that a full machine concept meeting the high-level requirements can be developed. Once that is in place, the elements of machine design which may prevent the alternative technology from meeting its initial promises can be identified and the full research effort required to fully realise the benefits can be assessed. In general, fusion power plant design is highly integrated and no one breakthrough gives great benefits as other performance-limiting factors come rapidly into play.

An appropriate work-flow may be: the proponents of a new technology outline the development pathway, the claimed benefits, evidence, and additional time and cost for development. A rapid and simplified system evaluation can then take place to identify the overall benefits and potential show-stoppers, allowing a cost benefit evaluation of the proposal. This can then be used to identify routes to further development (and the development of other affected systems) and the evidence base required for its incorporation into the baseline.

\section{Conclusions}

The EU-DEMO baseline remains the standard reference point for integrating the European fusion research programme, but does not represent a final conceptual design and will change as research results and technological developments are further incorporated. The emphasis is on the development of power plant design capabilities by focussing on real design problems and the production of flexible tools and systems that allow efficient design point development as technological downselection occurs en route to a final conceptual design. To ensure that the design space available is fully evaluated along this pathway, with technology and physics options consistent with the EUROfusion roadmap timescales, alternative concepts are explored to assess their potential benefits and costs.

In this case it appears that the most promising alternative magnetic configuration is the snowflake divertor, but much more development of the concept and demonstration of its ability to handle high exhaust powers is required before it can be incorporated into power plant designs seriously. Super-X designs suffer from major foreseeable integration issues and will require extensive development of supporting technology such as a divertor cassette with integrated coils or similar that is efficiently remote handleable. In addition, a super- $X$ is effective in mitigating heat flux to the divertor for all strike points only in double-null configurations, substantially increasing the magnetised volume of the machine. This configuration also requires poloidal field coils inside the toroidal field coils, raising significant feasibility and assembly issues. It is also the case that divertor performance in these configurations contains a great many unknowns that current models cannot illuminate. Experiments calibrating and extending SoL and divertor physics are needed to provide confidence in these designs and support the development of power plant concepts incorporating them.

\section{Acknowledgements}

This work has been carried out within the framework of the EUROfusion Consortium and has received funding from the Euratom research and training programme 2014-2018 under grant agreement No 633053 and from the RCUK Energy Programme [grant number EP/ I501045]. The views and opinions expressed herein do not necessarily reflect those of the European Commission.

\section{References}

[1] F. Romanelli, A roadmap to the realisation of fusion energy, Tech. rep., EFDA (2012).

[2] A. C. C. Sips, et al., Progress in preparing scenarios for ITER operation, Tech. Rep. EFDA-JET-CP(14)06/38, EFDA-JET (2014).

[3] H. Zohm, et al., A stepladder approach to a tokamak fusion power plant, Nuclear Fusion 57 (086002).

[4] P. Bruzzone, et al., LTS and HTS high current conductor development for DEMO, Fusion Engineering and Design 96-97 (2015) 77-82.

[5] M. Kotschenreuther, P. M. Valanju, S. M. Mahajan, J. C. Wiley, On heat loading, novel divertors, and fusion reactors, Physics of Plasmas 14 (2007) 072502.

[6] D. D. Ryotov, V. A. Soukhanovskii, The snowflake divertor, Physics of Plasmas 22 (2015) 110901.

[7] S. McIntosh, et al., A DEMO relevant long leg divertor with external poloidal field coils, Tech. Rep. EUROFUSION WPDTT1-CP(16) 15498 , EUROfusion (2016).

[8] R. Ambrosino, et al., Final report on activity DTT1-AC3: Equilibrium generation, Tech. Rep. EFDA-D-2MV7L9, EUROfusion (2016).

[9] M. Kovari, R. Kemp, H. Lux, P. J. Knight, J. Morris, D. J. Ward, "process": A systems code for fusion power plants - part 1: Physics, Fusion Engineering and Design 89 (12) (2014) 3054-3069.

[10] O. Crofts, et al., Overview of progress on the European DEMO remote maintenance strategy, Tech. Rep. EUROFUSION CP(15)06/18, EUROfusion (2015). 